\title{
Upgrading of aqueous bioethanol to higher alcohols over NiSn/MgAlO catalyst
}

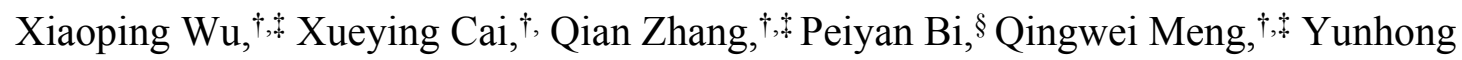

$$
\mathrm{Pi}^{\dagger}, \stackrel{\dagger}{\mathrm{t}} \text { and Tiejun Wang* }{ }^{*}, \dagger
$$

¡School of Chemical Engineering and Light Industry, Guangdong University of Technology, Guangzhou 510006, China

${ }^{\ddagger}$ Guangzhou Key Laboratory of Clean Transportation Energy Chemistry, Guangzhou 510006, China

§Jiangxi Province Key Laboratory of Polymer Micro/Nano Manufacturing and Devices, School of Chemistry, Biology and Materials Science, East China University of Technology, Nanchang 330013,

China

Corresponding author at: School of Chemical Engineering and Light Industry, Guangdong University of Technology, Guangzhou 510006, China.

E-mail address: tjwang@gdut.edu.cn (T. Wang).

Number of pages: 11 (including cover);

Number of Schemes: 1;

Number of Figures: 6;

Number of Tables: 3 . 
Scheme S1. Mechanism diagram of catalytic conversion of small alcohols to higher alcohols through Guerbet reaction. $\left(\mathrm{R}=\mathrm{H}, \mathrm{CH}_{3} \ldots\right)$

$$
\begin{aligned}
& \text { Step 1: } \mathrm{RCH}_{2} \mathrm{CH}_{2} \mathrm{OH} \stackrel{\text { Dehydrogenation }}{\stackrel{\text { Metal active sites }}{\longrightarrow}} \mathrm{RCH}_{2} \mathrm{CHO}+\mathrm{H}_{2} \\
& \text { Step 2: } \mathrm{RCH}_{2} \mathrm{CHO}+\mathrm{RCH}_{2} \mathrm{CHO} \underset{\mathrm{R}}{\stackrel{\text { Aldol condensation }}{\longrightarrow}} \underset{\text { Base sites }}{\mathrm{RCH}} \mathrm{CH}=\underset{\mathrm{T}}{\mathrm{CCHO}}+\mathrm{H}_{2} \mathrm{O}
\end{aligned}
$$

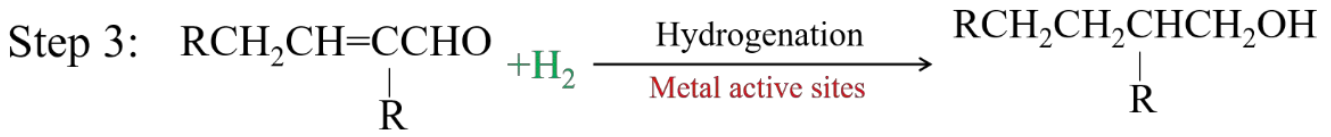




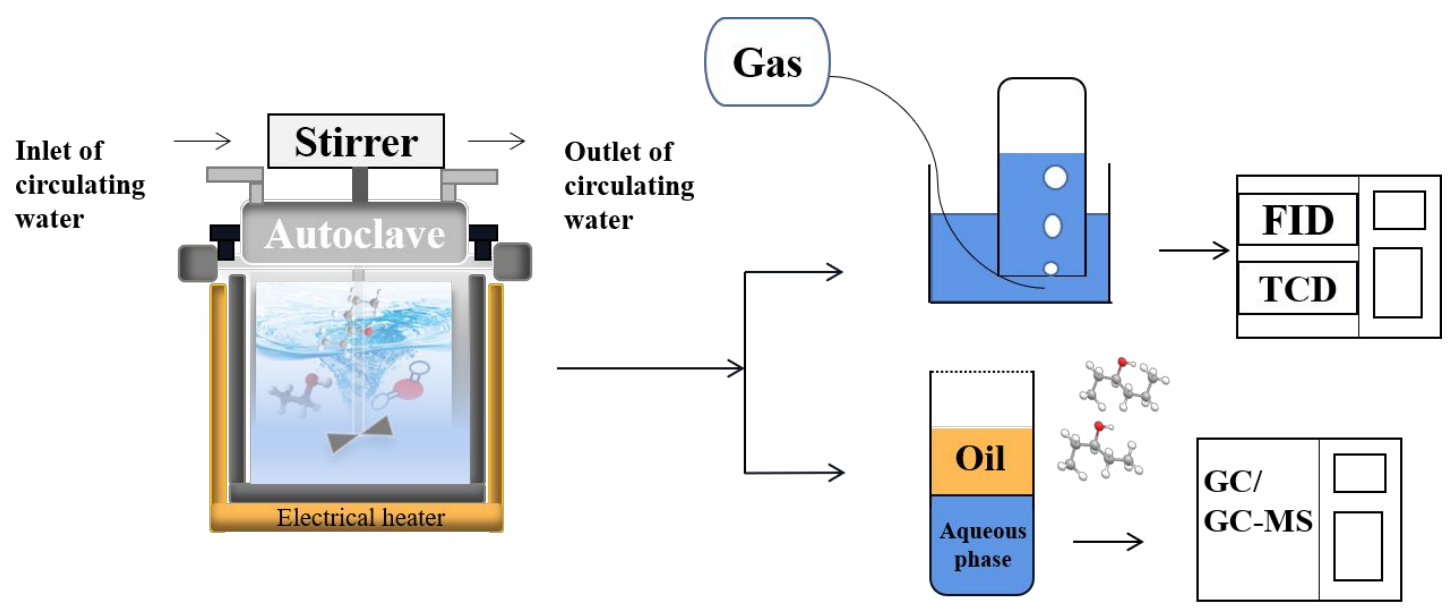

Figure S1. Schematic diagram of the experimental setup for the aqueous ethanol upgrading to higher alcohols. 


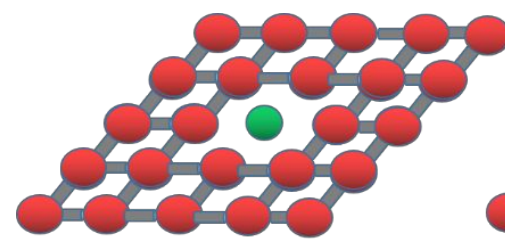

(a)

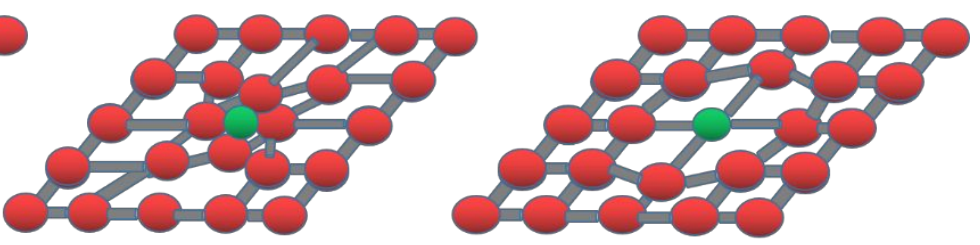

(b)

(c)

$\mathrm{Mg}^{2+} \bigcirc \mathrm{Ni}^{2+}$

Figure S2. Simulates the schematic diagram of the latticle defects caused by partial substitution of $\mathrm{Mg}^{2+}$ by $\mathrm{Ni}^{2+}$ in the structure of $\mathrm{MgAl}$ hydrotalcite. (a) $\mathrm{Ni}$ atoms in the crystal squeeze into the gap between the nodes; (b) and (c): Ni atoms in the crystal replace some $\mathrm{Mg}$ atoms. 

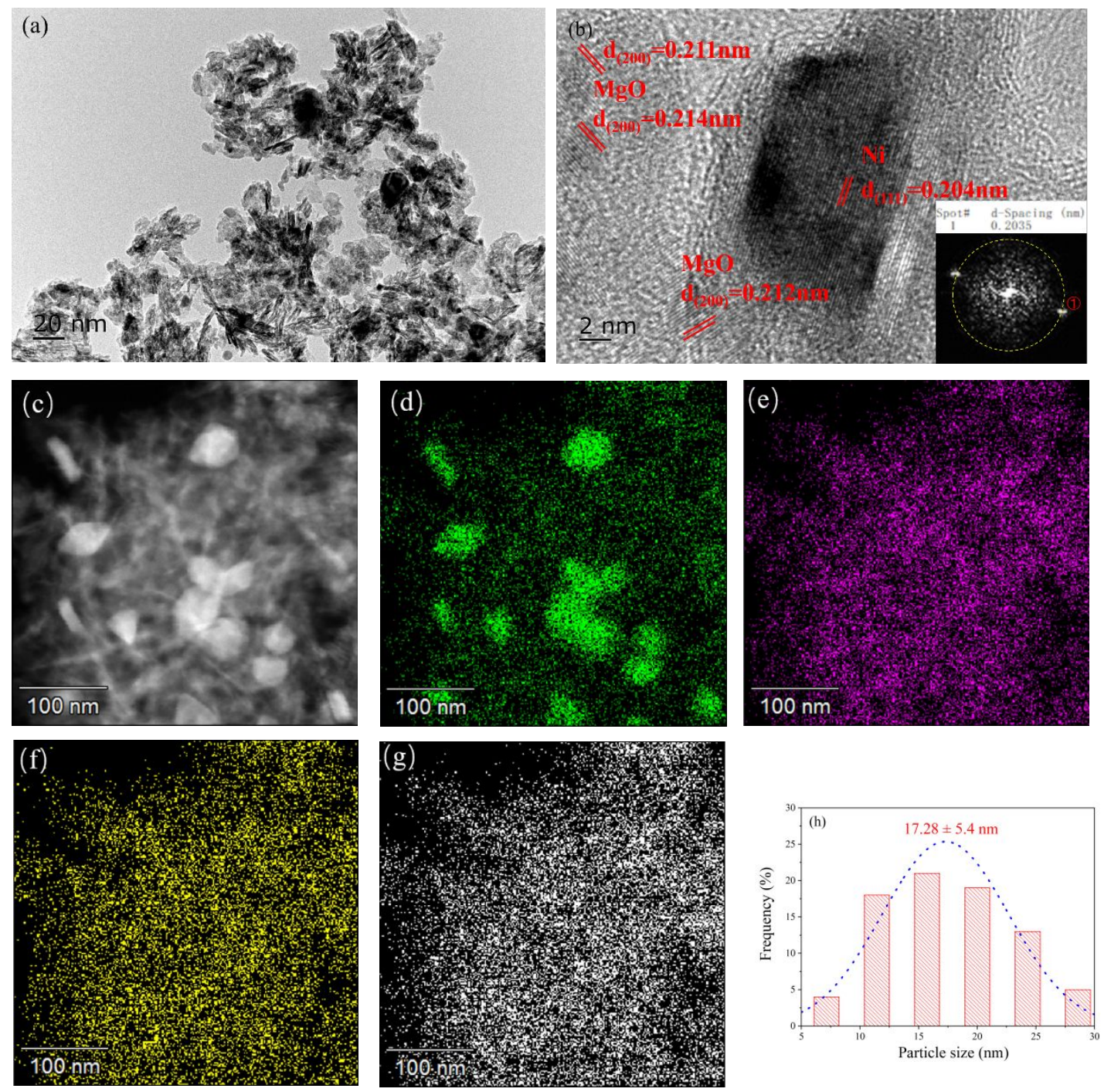

Figure S3. (a) TEM, (b) HRTEM with FFT of electron diffraction data, and (c) HAADF-STEM images of the freshly reduced Ni/MgAlO catalyst; EDX element mapping images of (d) $\mathrm{Ni}$, (e) $\mathrm{Mg}$,(f) $\mathrm{Al}$, and (g) O. (h) Size distribution of metal particles. 

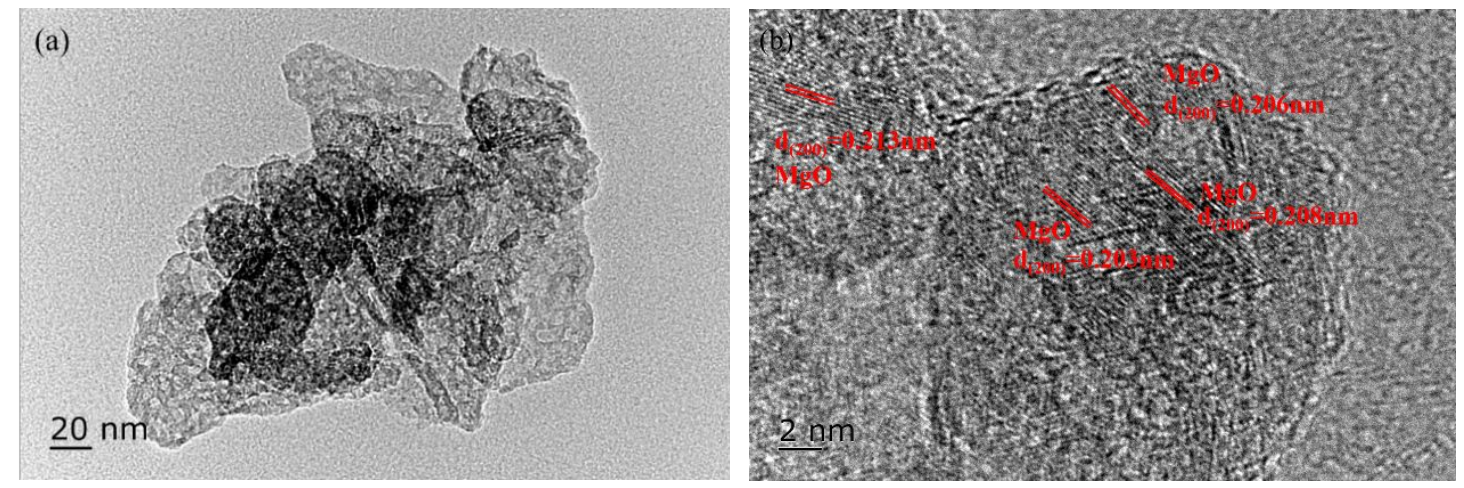

Figure S4. (a) TEM and (b) HRTEM images of the freshly reduced $\mathrm{Sn} / \mathrm{MgAlO}$ catalyst. 

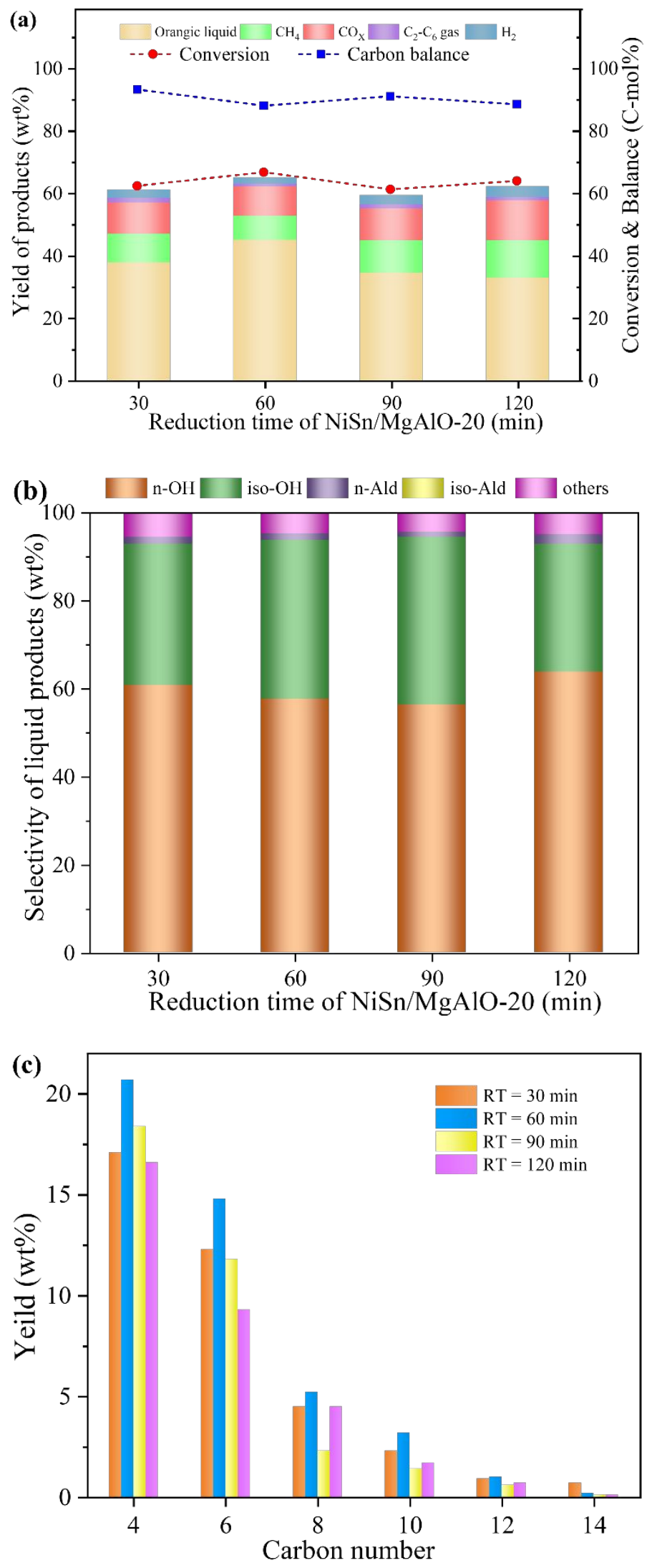

Figure S5. (a) Ethanol conversion, yield of products and carbon balance, 
(b) Selectivity of liquid products, (c) Carbon distribution of liquid product in aqueous ethanol upgrading over $\mathrm{NiSn} / \mathrm{MgAlO}-20$ catalyst at various reduction time. (RT: reduction time of $\mathrm{NiSn} / \mathrm{MgAlO}-20$ catalyst, $\mathrm{CO}_{\mathrm{x}}: \mathrm{CO}$ and $\mathrm{CO}_{2}$, n-: normal, iso-: isomeric, $\mathrm{OH}$ : alcohol, Ald: aldehydes) 


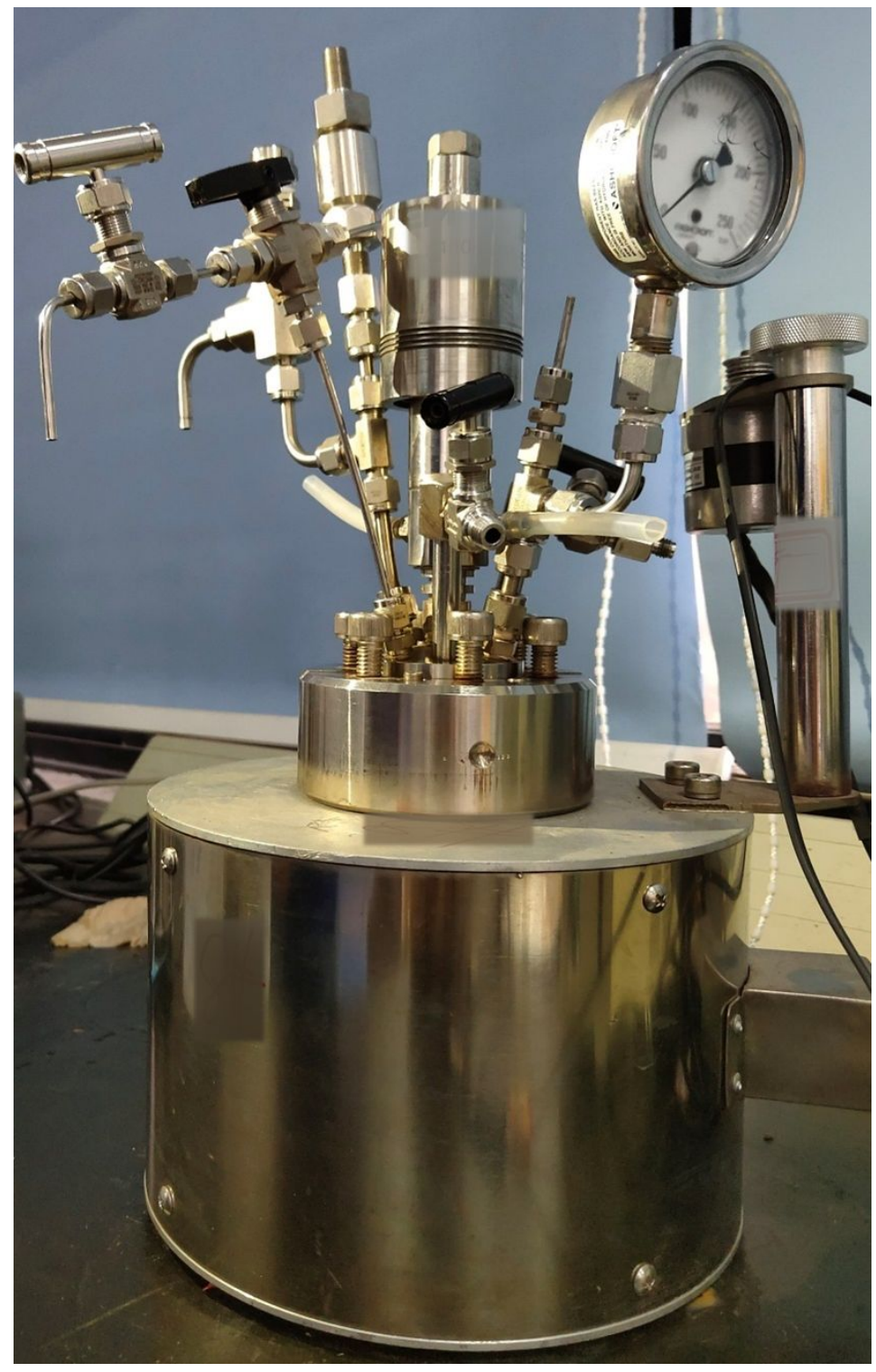

Figure S6. A photo of a stainless steel micro autoclave that can be sampled online. 
Table S1. Summary of binding energy and surface content of Ni over different catalysts.

\begin{tabular}{|c|c|c|c|c|c|c|}
\hline \multirow[b]{2}{*}{ Catalyst } & \multicolumn{3}{|c|}{ Binding energy $(\mathrm{eV})$} & \multicolumn{3}{|c|}{ Content (atomic\%) } \\
\hline & $\mathrm{Ni}^{0}$ & $\mathrm{Ni}^{2+}$ & $\mathrm{Ni}^{\delta+}$ & $\mathrm{Ni}^{0}$ & $\mathrm{Ni}^{2+}$ & $\mathrm{Ni}^{i^{\delta+}}$ \\
\hline Fresh Ni-MgAlO & 852.8 & 854.6 & 855.8 & 7.4 & 15.8 & 76.8 \\
\hline Fresh NiSn-MgAlO & 852.1 & 854.7 & 855.4 & 9.6 & 6.7 & 83.7 \\
\hline Spent NiSn-MgAlO & 852.3 & 855.0 & 855.5 & 16.6 & 13.9 & 69.5 \\
\hline
\end{tabular}

Table S2. Summary of binding energy and surface content of Sn over different catalysts.

\begin{tabular}{lccccccc}
\hline & \multicolumn{1}{c}{$\begin{array}{c}\text { Chemical } \\
\text { state }\end{array}$} & \multicolumn{3}{c}{ Binding energy (eV) } & & \multicolumn{3}{c}{ Content (atomic\%) } \\
\cline { 3 - 5 } \cline { 6 - 8 } Catalyst & $\mathrm{Sn}^{0}$ & $\mathrm{Sn}^{2+}$ & $\mathrm{Sn}^{4+}$ & & $\mathrm{Sn}^{0}$ & $\mathrm{Sn}^{2+}$ & $\mathrm{Sn}^{4+}$ \\
\hline Fresh NiSn-MgAlO & 484.8 & 486.1 & 486.6 & & 7.6 & 7.6 & 84.8 \\
Spent NiSn-MgAlO & 484.8 & 486.0 & 486.5 & & 16.1 & 14.0 & 69.9 \\
\hline
\end{tabular}


Table S3. Ethanol upgrading over the NiSn-MgAlO catalysts at various molar ratio of $\mathrm{Ni} / \mathrm{Sn}^{\mathrm{a}}$.

\begin{tabular}{llllllll}
\hline $\mathrm{Ni} /$ Sn ratio & Blank $^{\mathrm{b}}$ & $0: 1$ & $10: 1$ & $20: 1$ & $30: 1$ & $40: 1$ & $1: 0$ \\
\hline Conversion (C-mol\%) & 7.4 & 26.7 & 56.4 & 66.9 & 64.9 & 67.7 & 89.8 \\
$Y_{\text {liquid }}$ (C-mol\%) & 2.2 & 25.1 & 28.9 & 48.4 & 38.9 & 36.9 & 37.2 \\
Balance (C-mol\%) & 91.1 & 92.7 & 88.6 & 88.2 & 95.2 & 84.6 & 93.8 \\
$Y_{\text {products }}$ (wt\%) & & & & & & & \\
Organic liquid & 1.4 & 17.1 & 26.7 & 45.1 & 35.5 & 33.7 & 32.7 \\
$\mathrm{CH}_{4}$ & 3.1 & 0.2 & 16.3 & 7.7 & 13.2 & 14.5 & 34.9 \\
$\mathrm{CO}_{\mathrm{x}}$ & 0.4 & u.d. & 9.3 & 9.4 & 10.2 & 11.2 & 13.0 \\
$\mathrm{C}_{2}$-C $\mathrm{C}_{6}$ gas & 0.2 & 5.2 & 2.5 & 0.9 & 1.6 & 1.7 & 3.6 \\
$\mathrm{H}_{2}$ & 1.2 & 4.2 & 5.3 & 1.9 & 2.9 & 3.3 & 7.1 \\
Selectivity of liquid products (\%) & & & & & & \\
$\mathrm{n}-\mathrm{OH}$ & 100.0 & 75.7 & 67.7 & 57.7 & 58.1 & 67.4 & 58.9 \\
iso-OH & u.d. & 19.4 & 26.3 & 36.1 & 34.3 & 25.7 & 21.5 \\
$\mathrm{n}$-Ald & u.d. & u.d. & 1.5 & 1.5 & 2.4 & 2.0 & 15.9 \\
unidentified & u.d. & 4.8 & 4.5 & 4.7 & 5.2 & 4.9 & 3.7 \\
Carbon distribution (\%) & & & & & & & \\
4 & $100.0^{c}$ & 10.2 & 13.0 & 20.7 & 17.4 & 16.2 & 16.9 \\
6 & u.d. & 5.8 & 5.3 & 14.8 & 13.4 & 12.5 & 10.5 \\
8 & u.d. & 1.1 & 3.0 & 5.2 & 4.4 & 4.6 & 4.6 \\
10 & u.d. & u.d. & 1.4 & 3.2 & 2.5 & 2.5 & 2.6 \\
12 & u.d. & u.d. & u.d. & 1.0 & 0.7 & 0.6 & 1.6 \\
14 & u.d. & u.d. & u.d. & 0.2 & 0.5 & 0.5 & 1.0 \\
\hline
\end{tabular}

a Reaction condition: 0.6 g catalyst, $250^{\circ} \mathrm{C}, 1 \mathrm{~g} \mathrm{NaOH}, 10 \mathrm{~g} \mathrm{H}_{2} \mathrm{O}, 10 \mathrm{~g}$ ethanol, $12 \mathrm{~h}$. (Y: yield, n-: normal, iso-: isomeric, OH: alcohol, Ald: aldehydes, u.d.: undetected)

${ }^{b}$ Blank control experiment: the experiment condition is same as the typical conditions except without catalyst.

${ }^{\mathrm{c}}$ No spontaneously separated oil-phase was collected, the only distinguishable product is the traces amount of butanol which dissolved in water-phase due to the sparing solubility in water. 